$<$ Supporting Information>

\title{
Metal Ion Sensing Novel Calix[4]crown Fluoroionophore with Two-Photon Absorption Property
}

\author{
Jong Seung Kim ${ }^{1, *}$ Hyun Jung Kim, ${ }^{1}$ Hwan Myung Kim, ${ }^{2}$ Su Ho Kim, ${ }^{1}$ Jeong Won Lee, ${ }^{1}$ \\ Sung Kuk Kim, ${ }^{1}$ and Bong Rae $\mathrm{Cho}^{2, *}$ \\ ${ }^{1}$ Department of Chemistry, Institute of Nanossensor \& Biotechnology, Dankook University, Seoul 140-714, \\ Korea \\ ${ }^{2}$ Department of Chemistry, Korea University, Seoul 136-701, Korea \\ * Corresponding authors: Jong Seung Kim (jongskim@ dankook.ac.kr); Bong Rae Cho (hobr@korea.ac.kr)
}

Figure S1 page $\mathrm{S} 2$

Figure S2 page $\mathrm{S} 2$

Figure S3 page $\mathrm{S} 3$

Figure S4 page $\mathrm{S} 3$

Figure S5 page $\mathrm{S} 4$

Figure S6 page $\mathrm{S} 4$

Figure S7 page S5

Figure S8 page S5

Figure S9 page $S 6$

Data S10 page S6 

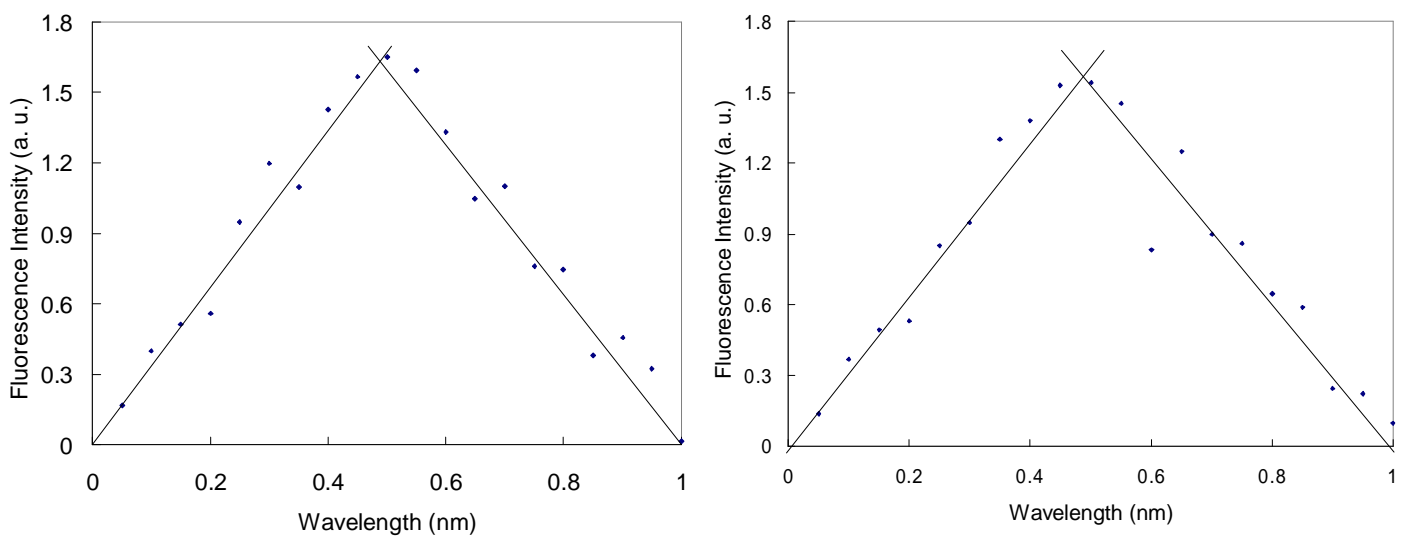

Figure S1. Job plots for $\mathbf{1} \cdot \mathrm{Al}^{3+}$ (left) and $\mathbf{1} \cdot \mathrm{Pb}^{2+}$ (right).

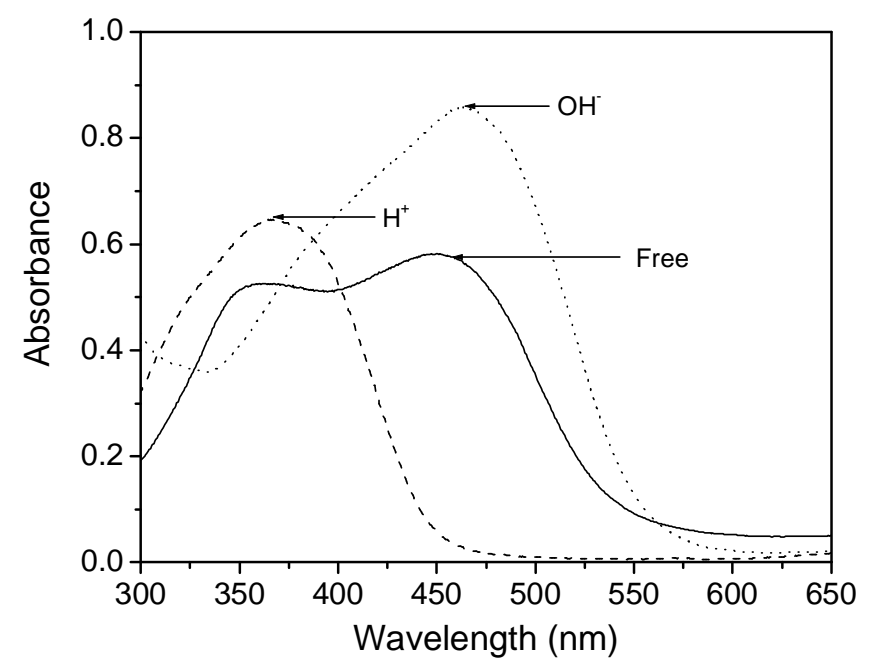

Figure S2. UV-vis spectra of $\mathbf{1}$ upon the addition of acid and base. Conditions: $\mathbf{1}$ $(0.01 \mathrm{mM}) / \mathrm{CH}_{3} \mathrm{CN}: \mathrm{CHCl}_{3}(3: 1)$; acid and base 500 equiv $(10 \mu \mathrm{M}) / \mathrm{CH}_{3} \mathrm{CN}$. 


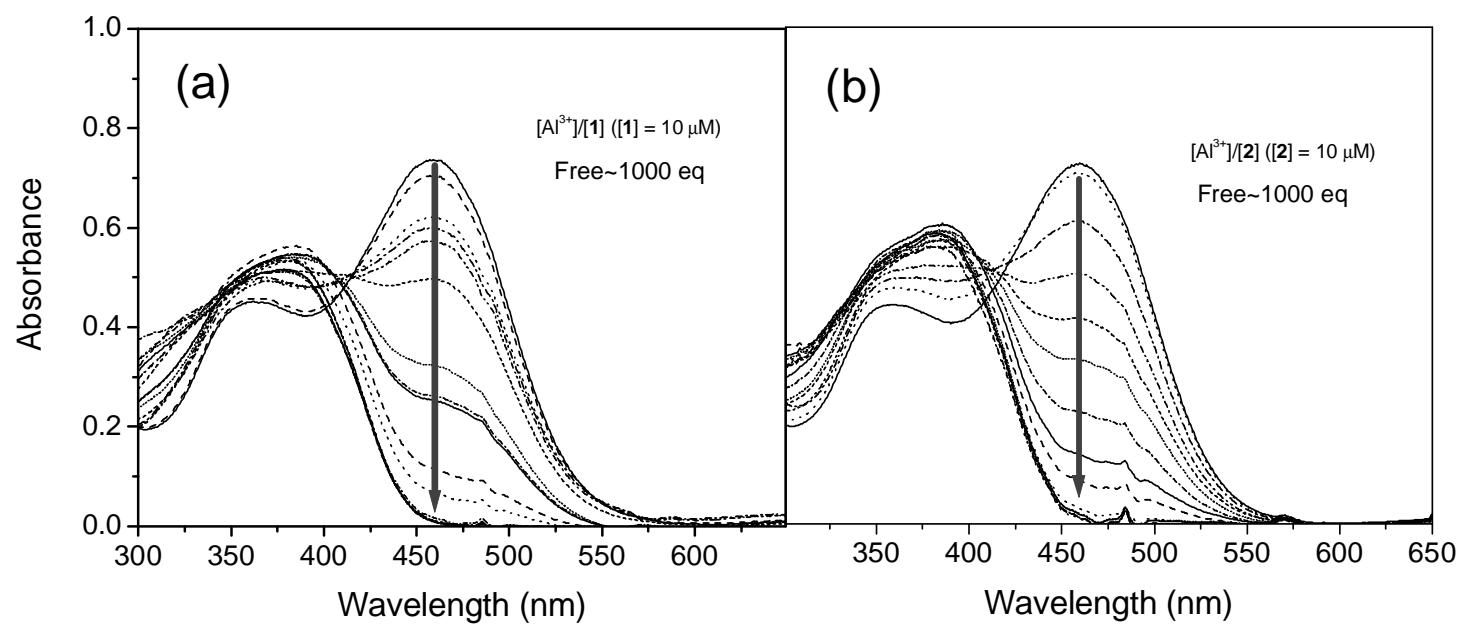

Figure S3. UV-vis spectra of (a) 1 (b) 2 upon the addition of various amounts of $\mathrm{Al}^{3+}$ in $\mathrm{MeCN}$.

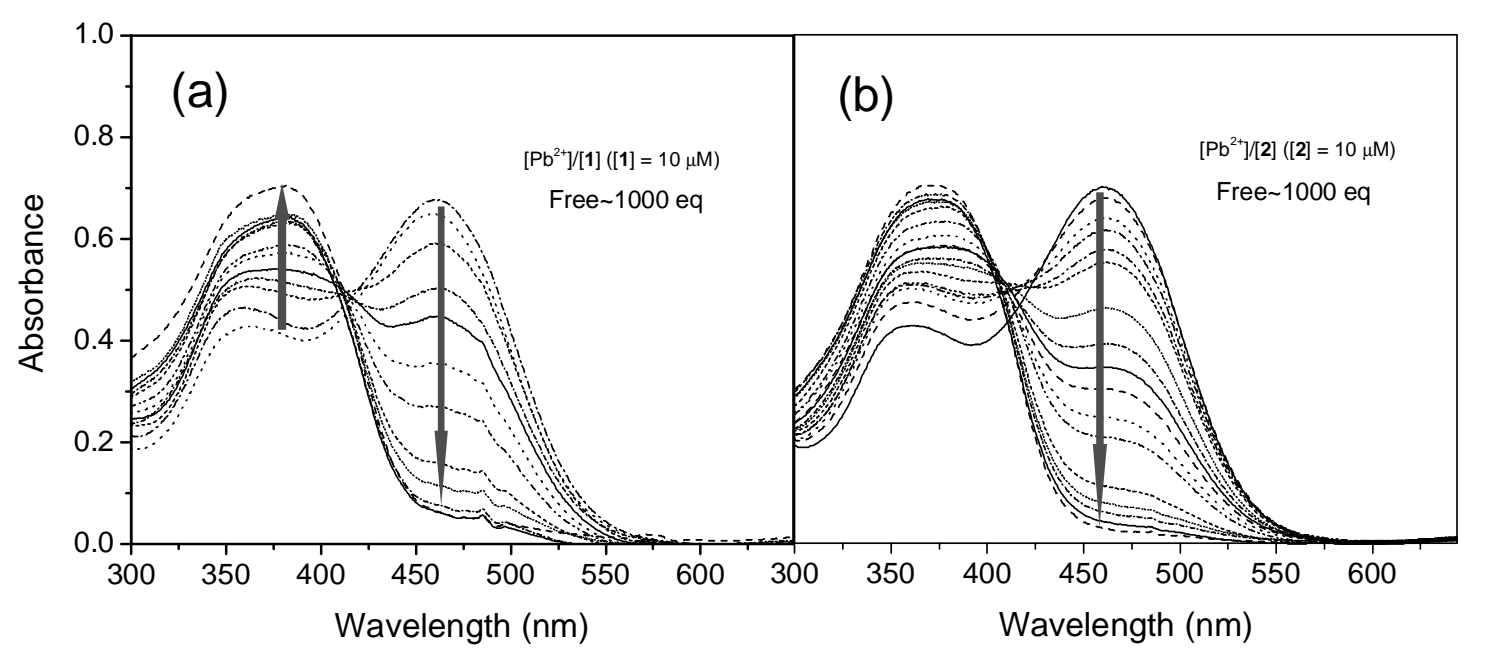

Figure S4. UV-vis spectra of (a) 1 (b) 2 upon the addition of various amounts of $\mathrm{Pb}^{2+}$ in $\mathrm{MeCN}$. 


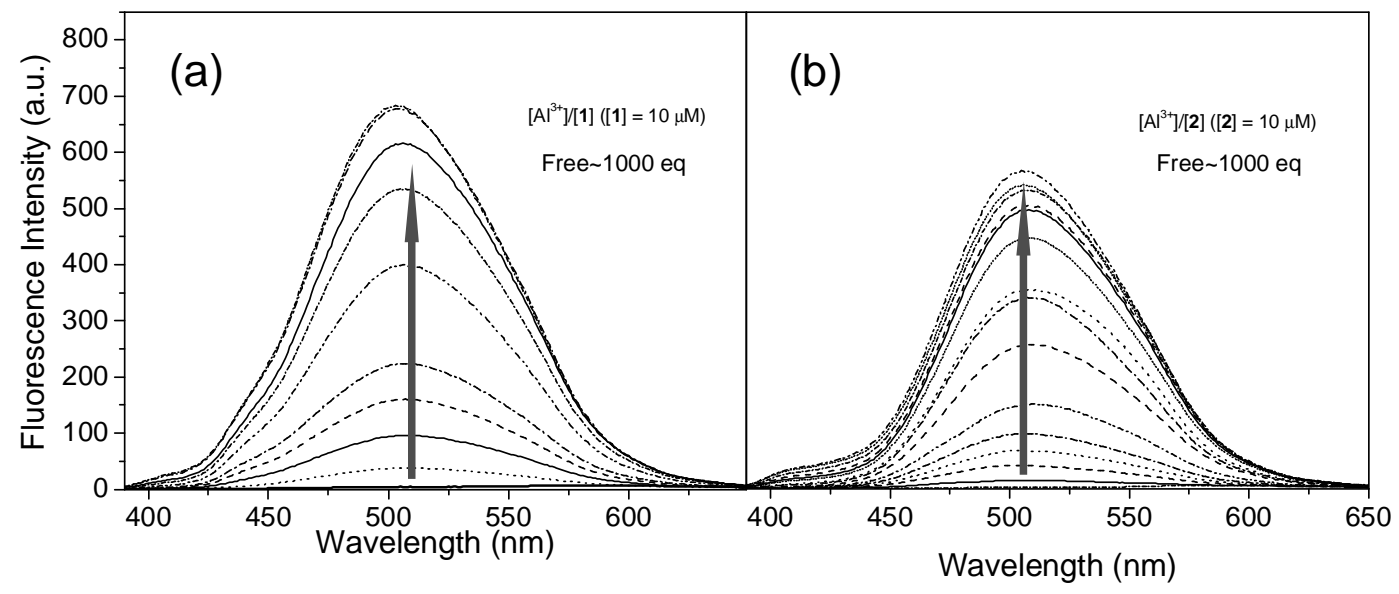

Figure S5. Fluorescence emission spectra of (a) 1 (b) 2 upon the addition of various amounts of $\mathrm{Pb}^{2+}$ in $\mathrm{MeCN}$. The excitation wavelength is $385 \mathrm{~nm}$.

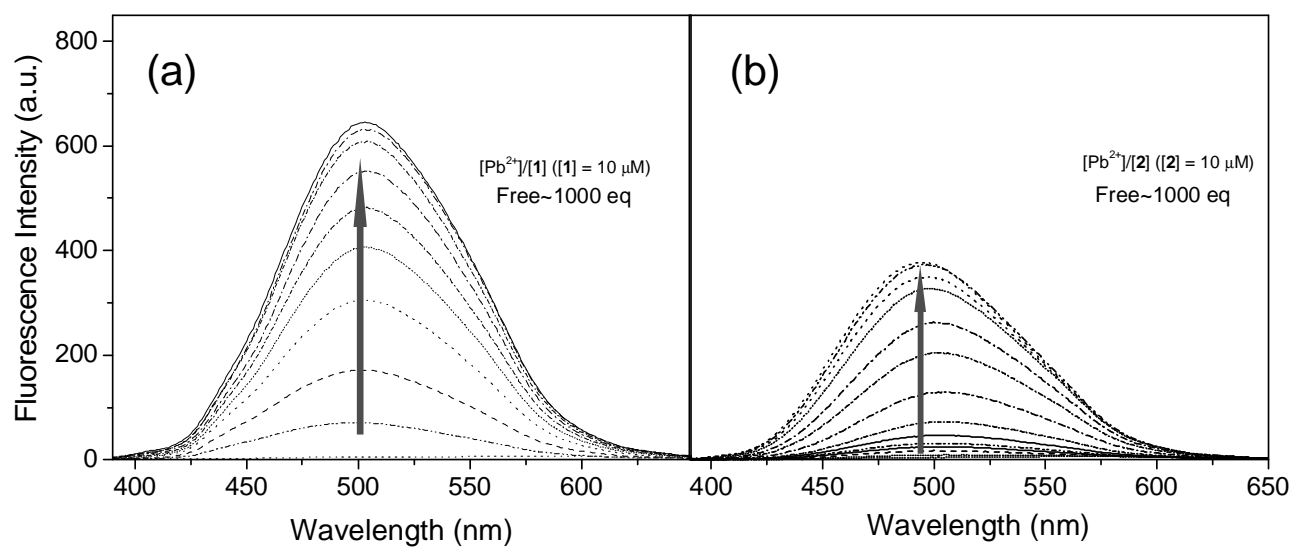

Figure S6. Fluorescence emission spectra of (a) 1 (b) 2 upon the addition of various amounts of $\mathrm{Pb}^{2+}$ in $\mathrm{MeCN}$. The excitation wavelength is $385 \mathrm{~nm}$. 


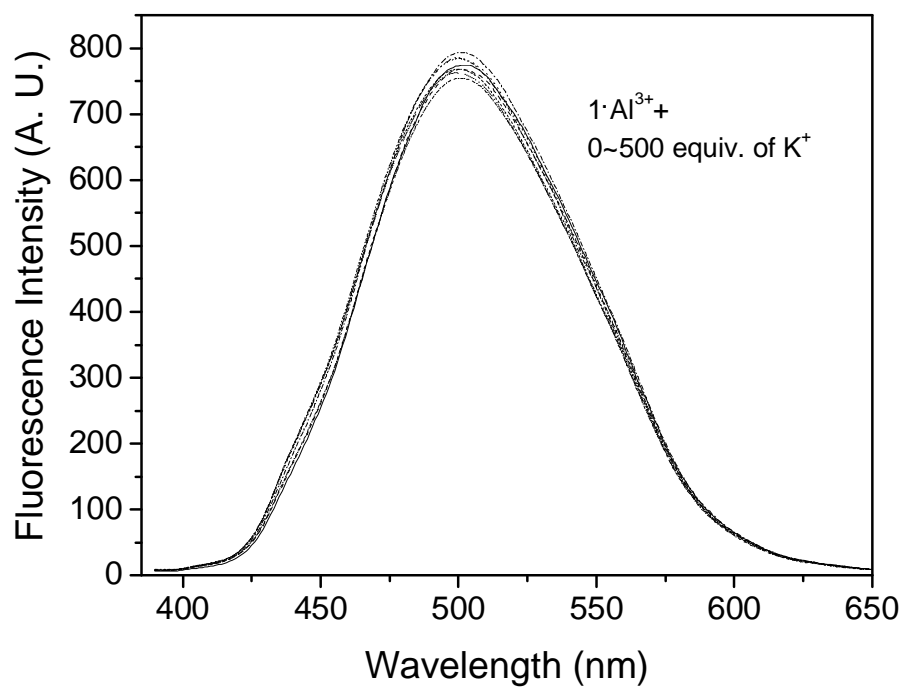

Figure S7. Fluorescence changes spectra of $\mathbf{1}(4.0 \mu \mathrm{M})$ with $\mathrm{K}^{+}$titration in the presence of $\mathrm{Al}^{3+}$. The excitation wavelength is $385 \mathrm{~nm}$.

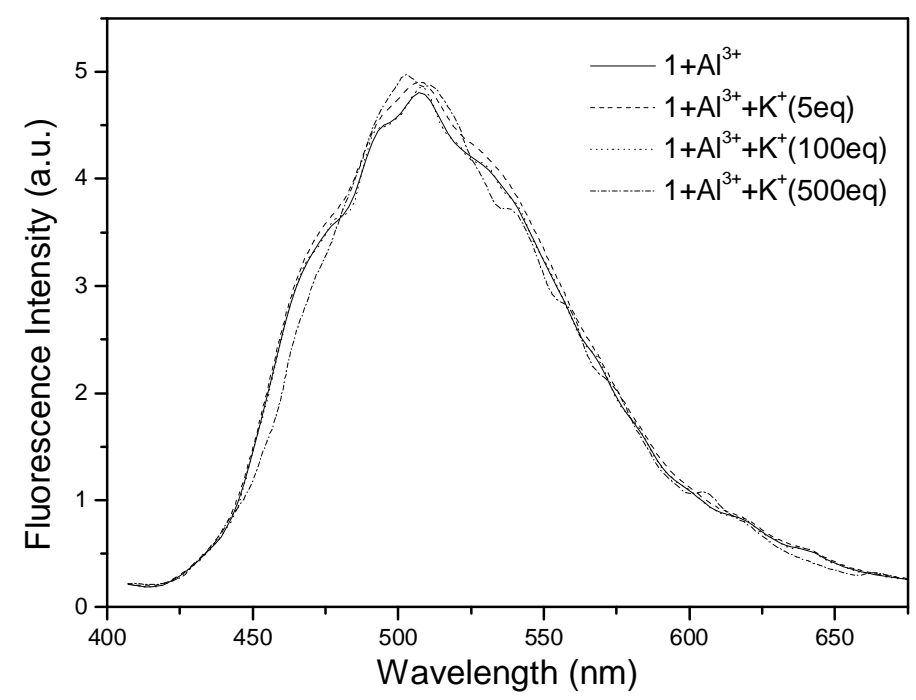

Figure S8. Two-photon fluorescence changes spectra of $1(4.0 \mu \mathrm{M})$ with $\mathrm{K}^{+}$titration in the presence of $\mathrm{Al}^{3+}$. The excitation wavelength is $780 \mathrm{~nm}$. 


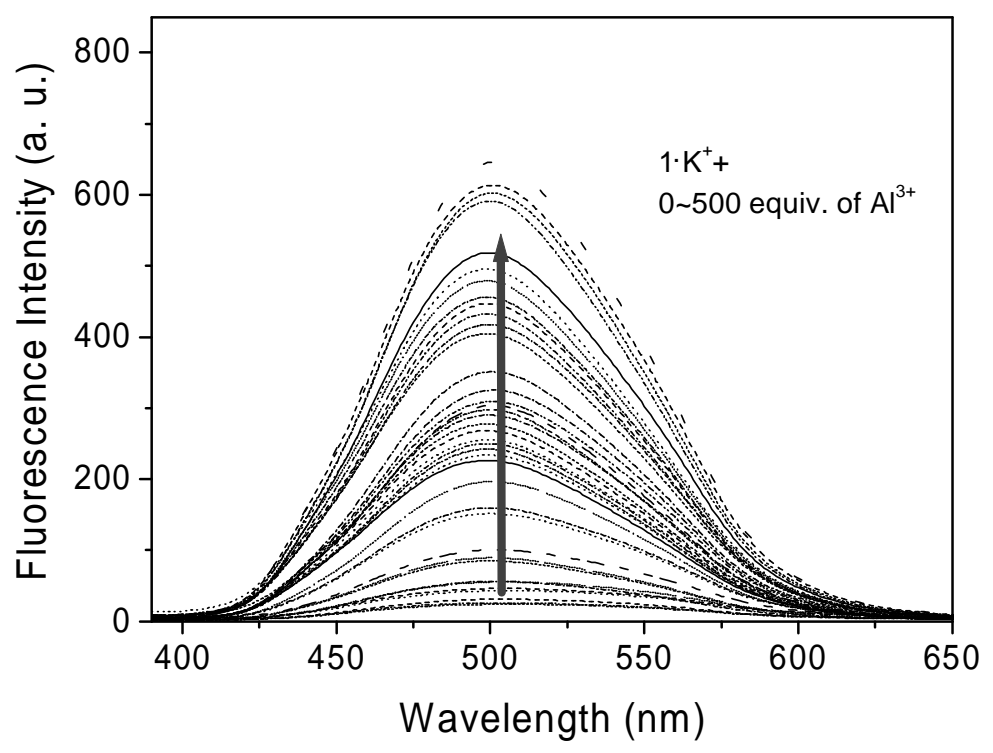

Figure S9. Fluorescence changes spectra of $\mathbf{1}(4.0 \mu \mathrm{M})$ with $\mathrm{K}^{+}$titration in the presence of $\mathrm{Al}^{3+}$. The excitation wavelength is $385 \mathrm{~nm}$.

\section{NMR Data S10}

25,27-Bis[ $N$-(1-((2,5-dicyano-(1-(p-dipentylamino)styryl-4-styryl)phenyl) aminocarbonyl)methoxy] calix[4] crown-5 in the 1,3-alternate conformation (1).

${ }^{1} \mathrm{H}$ NMR (200 MHz, $\mathrm{CDCl}_{3}$ ): $\delta 8.34$ (br s, $2 \mathrm{H}, \mathrm{NH}$ ), 7.92 (s, $\left.2 \mathrm{H}, \mathrm{ArH}\right), 7.49-7.02(\mathrm{~m}, 14 \mathrm{H}$, $\mathrm{ArH} ; 12 \mathrm{H}, \mathrm{ArH}$, calix[4]arene; $8 \mathrm{H}, \mathrm{CH}=\mathrm{CH}$ ), 6.59-6.68 (dd, $4 \mathrm{H}, \mathrm{Ar} H, J=7.60 \& 7.60$ $\mathrm{Hz}), 3.91$ (s, $8 \mathrm{H}, \mathrm{ArCH}_{2} \mathrm{Ar}$ ), 3.81 (s, $4 \mathrm{H}, \mathrm{ArOCH}_{2} \mathrm{CO}$ ), 3.64 (br s, $12 \mathrm{H}, \mathrm{OCH}_{2} \mathrm{CH}_{2} \mathrm{O}$ ), 3.3 (br s, $4 \mathrm{H}, \mathrm{OCH}_{2} \mathrm{CH}_{2} \mathrm{O} ; 8 \mathrm{H}$., $\mathrm{NCH}_{2} \mathrm{CH}_{2}$ ), 1.61 (br s, $8 \mathrm{H}, \mathrm{CH}_{2} \mathrm{CH}_{2} \mathrm{CH}_{2}$ ), 1.34 (br s, $16 \mathrm{H}$, $\left.\mathrm{CH}_{2} \mathrm{CH}_{2} \mathrm{CH}_{3}\right), 0.90\left(\mathrm{t}, 12 \mathrm{H}, \mathrm{CH}_{2} \mathrm{CH}_{2} \mathrm{CH}_{3}, \mathrm{~J}=6.6 \mathrm{~Hz}\right) .{ }^{13} \mathrm{C} \mathrm{NMR}\left(50 \mathrm{MHz}, \mathrm{CDCl}_{3}\right): 168.5$, $157.4,156.3,150.0,140.1,139.1,138.0,136.3,135.8,135.2,134.8,134.6,134.1,132.8$, $131.6,130.9,130.2,129.7,128.9,125.4,124.8,123.4,121.9,121.3,117.9,116.9,115.6$, $114.8,112.4,73.6,72.5,71.9,70.8,69.8,52.0,38.9,30.3,28.0,23.6,15.1 \mathrm{ppm}$.

25,27-Bis[ $N$-(1-((2,5-dicyano-(1-(p-dipentylamino)styryl-4-styryl)phenyl)aminocarbonyl)methoxy]-26,28-dipropyloxy-calix[4]arene in the 1,3-alternate conformation (2). ${ }^{1} \mathrm{H}$ NMR $\left(200 \mathrm{MHz}, \mathrm{CDCl}_{3}\right): \delta 8.45$ (br s, $\left.2 \mathrm{H}, \mathrm{NH}\right), 8.00-6.55(\mathrm{~m}, 32 \mathrm{H}, \mathrm{ArH} ; 8 \mathrm{H}$, $\mathrm{CH}=\mathrm{CH}$ ), 3.74-3.65 (m, $8 \mathrm{H}, \mathrm{ArCH}_{2} \mathrm{Ar} ; 4 \mathrm{H}, \mathrm{ArOCH}_{2} \mathrm{CH}_{2} ; 4 \mathrm{H}, \mathrm{ArOCH}_{2} \mathrm{CO}$ ), 3.31 (br s, 8 H., $\mathrm{NCH}_{2} \mathrm{CH}_{2}$ ), 1.62 (br s, $8 \mathrm{H}, \mathrm{CH}_{2} \mathrm{CH}_{2} \mathrm{CH}_{2}$ ), 1.37 (br s, $16 \mathrm{H}, \mathrm{CH}_{2} \mathrm{CH}_{2} \mathrm{CH}_{3}$ ), 0.95 (broad s, $\left.12 \mathrm{H}, \mathrm{CH}_{2} \mathrm{CH}_{2} \mathrm{CH}_{3}\right) .{ }^{13} \mathrm{C}$ NMR $\left(50 \mathrm{MHz}, \mathrm{CDCl}_{3}\right): 168.5,157.4,156.3,150.0,140.1,139.1$, 138.0, 136.3, 135.8, 135.2, 134.8, 134.6, 134.1, 132.8, 131.6, 130.9, 130.2, 129.7, 128.9, $125.4,124.8,123.4,121.9,121.3,117.9,116.9,115.6,114.8,112.4,73.6,72.5,71.9,70.8$, 
69.8, 52.0, 38.9, 30.3, 28.0, 23.6, $15.1 \mathrm{ppm}$.

2,5-Dicyano-[1-( $p$-dipentylamino)styryl-4-( $p$-amino)styryl]benzene (3).

${ }^{1} \mathrm{H}$ NMR $\left(300 \mathrm{MHz}, \mathrm{CDCl}_{3}\right): \delta 7.94(\mathrm{~s}, 1 \mathrm{H}), 7.91(\mathrm{~s}, 1 \mathrm{H}), 7.43(\mathrm{~d}, 2 \mathrm{H}, J=9.0 \mathrm{~Hz}), 7.39$ $(\mathrm{d}, 2 \mathrm{H}, J=9.0 \mathrm{~Hz}), 7.20(\mathrm{~d}, 1 \mathrm{H}, J=18.0 \mathrm{~Hz}), 7.18(\mathrm{~d}, 1 \mathrm{H}, J=18.0 \mathrm{~Hz}), 7.11(\mathrm{~d}, 1 \mathrm{H}, J=$ $18.0 \mathrm{~Hz}), 7.08(\mathrm{~d}, 1 \mathrm{H}, J=18.0 \mathrm{~Hz}), 6.68(\mathrm{~d}, 2 \mathrm{H}, J=9.0 \mathrm{~Hz}), 6.62(\mathrm{~d}, 2 \mathrm{H}, J=9.0 \mathrm{~Hz})$, $3.91(\mathrm{~s}, 2 \mathrm{H}), 3.30$ (t, $4 \mathrm{H}, J=7.5 \mathrm{~Hz}$ ), 1.61 (quint, $4 \mathrm{H}, J=7.5 \mathrm{~Hz}), 1.35(\mathrm{~m}, 8 \mathrm{H}), 0.93$ (t, $6 \mathrm{H}, J=7.5 \mathrm{~Hz})$.

1-(p-Dipentylamino)styryl-4-(diethoxyphosphoryl)methyl-2,5-dicyanobenzene (5).

${ }^{1} \mathrm{H}$ NMR $\left(300 \mathrm{MHz}, \mathrm{CDCl}_{3}\right): \delta 7.99(\mathrm{~s}, 1 \mathrm{H}), 7.69(\mathrm{~d}, 1 \mathrm{H}, J=3.0 \mathrm{~Hz}), 7.43(\mathrm{~d}, 2 \mathrm{H}, J=9.0$ $\mathrm{Hz}), 7.22(\mathrm{~d}, 1 \mathrm{H}, J=18.0 \mathrm{~Hz}), 7.09(\mathrm{~d}, 1 \mathrm{H}, J=18.0 \mathrm{~Hz}), 6.62(\mathrm{~d}, 2 \mathrm{H}, J=9.0 \mathrm{~Hz}), 4.15$ $(\mathrm{m}, 4 \mathrm{H}), 3.36(\mathrm{~d}, 2 \mathrm{H}, J=21.0 \mathrm{~Hz}), 3.30(\mathrm{t}, 4 \mathrm{H}, J=7.5 \mathrm{~Hz}), 1.61$ (quint, $4 \mathrm{H}, J=7.5 \mathrm{~Hz}$ ), $1.35(\mathrm{~m}, 14 \mathrm{H}), 0.93(\mathrm{t}, 6 \mathrm{H}, J=7.5 \mathrm{~Hz})$. 